\section{Neutron Physics in the Taurides}

\author{
Alushta, URSS, 2-19 April 1974
}

The Second International School of Neutron Physics, organized by the Joint Institute for Nuclear Research in Dubna, was held in Alushta (The Crimea) from 2-19 April this year. The excellent hospitality and the high scientific standards of the meeting were the merit of a thoughtful organizing committee headed by Academician I.M. Frank. Minor incidents, like what was called "untypical Crimean weather", could not be blamed on the organizers!

Much could be said about the usefulness of meetings dedicated to different fields of science tied together by a common means of investigation. What follows is a brief outline of only a part of three weeks of intensive lecturing and discussion which covered most of the fields in nuclear, solid state and biophysics where neutron beams are used to investigate the properties of physical systems. During these three weeks, it became increasingly clear how much work remains to be done in the field of neutron physics. In particular, a number of new possibilities is being opened by the development of ultracold neutron sources. Thus the "star of the show" was, undoubtedly, the ultracold neutron, and much of the present report will be devoted to this subject.

Already in 1936 Fermi had shown that the ratio between the velocity of neutrons in a medium (v) and in vacuum $\left(v_{0}\right)$ could be expressed by an index of refraction

$$
\mathrm{n}^{2}=1-\lambda^{2} \frac{\mathrm{Nb}}{\pi}
$$

where $\lambda$ is the neutron wavelength, $\mathrm{N}$ the density of nuclei in the medium, and $b$ the neutron-nucleus scattering length. Given the (positive) values of $\mathrm{N}$ and $\mathrm{b}$, it appears from the above expression that for the majority of nuclei neutrons with $\lambda$ larger than, say, $500 \AA$, will have an imaginary index of refraction. As the energy of such neutrons is well below the average thermal energy, they are called ultracold neutrons.

The above phenomenon is formally analogous to the reflection of light from a metallic mirror. This means that neutrons with an energy $E$ less than a limiting value of

$$
\frac{2 \pi \hbar^{2}}{\mathrm{~m}} \mathrm{Nb} \approx 10^{-7} \mathrm{eV}
$$

can only penetrate into matter to a depth of about $\lambda$ and are essentially totally reflected for any incident angle. Hence, ultracold neutrons should not escape from a closed vessel but should remain inside either until their natural death by $\beta$-decay or until their absorption in the vessel walls (probability $\approx 10^{-4}-10^{-5}$ ).

Very few ultracold neutrons (only $10^{-12}$ of the total flux) come out from a reactor. Nevertheless, $10^{15}$ neutrons/ $\mathrm{cm}^{2} \cdot \mathrm{s}$ can be obtained from a highflux reactor, which still gives about $10^{3} \mathrm{UCN} / \mathrm{cm}^{2} \cdot \mathrm{s}$. Experiments in Dubna have confirmed that UCN can be contained in "bottles" for more than 100 seconds and UCN densities of 10 per litre have been obtained. It appears, however, that for instance, the obtained containment times are still about two orders of magnitude smaller than the maximum value predicted by theory. The reason for such a discrepancy is not yet totally understood but it is probably connected with absorption phenomena in the vessel walls.

What are ultracold neutrons good for? First, we finally have a "neutron bottle"; a dream dating from Fermi's times. Examples of the applications of such bottles were discussed in connection with two long-standing problems in nuclear physics: the neutron $\beta$-decay lifetime and the possible existence of a neutron electric-dipole moment (EDM).

Attempts to measure a neutron electric dipole moment generally involve the search for a small frequency shift. Aligned neutrons in a magnetic field rotate at the Larmor frequency. If they have a dipole moment $d$, the application of an electric field $\varepsilon$ non parallel to the neutron spin axis causes an additional torque and a slight shift in rotational frequency. The corresponding energy shift $\Delta \mathrm{E}=\mathrm{d} \cdot \varepsilon$ is related to the time $\Delta t$ a neutron spends in the vessel with the field, by the Heisenberg relation $\Delta \mathrm{E} \cdot \Delta \mathrm{t} \approx \hbar$. Classically speaking, the accuracy of the measurement depends on the amount of phase shift (relative to a clock operating at the Larmor frequency) which is accumulated while the neutrons are in the apparatus. With the use of neutron bottles the containment time of neutrons in the field and hence the accumulated phase shift can be significantly increased. As we have already mentioned, containment times $\Delta t$ of about $100 \mathrm{~s}$ could be obtained for ultracold neutrons, while classical experiments with a beam of slow neutrons gives only $\Delta \mathrm{t} \approx 0.01 \mathrm{~s}$. Thus the use of ultracold neutrons increases the sensitivity of the experiment by several orders of magnitude. With this in mind, the magnetic resonance spectrometer previously used to search for a neutron electric dipole moment at Oak Ridge was moved to the Laue-Langevin high-flux reactor in Grenoble. The cold moderator in Grenoble together with the higher reactor power will result in more than one thousand times the neutron intensity of the Oak Ridge experiment; first results indicate an upper limit for the neutron of $10^{-24}$ $\mathrm{cm}$ times the charge of the proton.

The interest in the existence of an EDM for the neutron is quite fundamental. The sensational discovery of $\mathrm{CP}$ violation in the decay of the neutral $\mathrm{K}_{2}^{\circ}$ meson implied a violation of time reversal invariance, since CPT is assumed to be conserved. The existence of an EDM for the neutron would provide the first direct evidence of $T$ violation.

The part of the school devoted to nuclear physics phenomena comprised a number of interesting reports of results based on more conventional techniques. Among these were a first-hand report on investigations of the parity non-conserving part of the nuclear force, a description of the reasons for fragmentation of singleparticle strength at higher excitation energies in nuclei, and some lively discussions of the mechanisms of direct $(n, \alpha)$ reactions and their possible implications for quartet structure in nuclei. Let it be said, however, that the choice of the topics to be described more extensively in this report was very much influenced by the fact that the authors were more impressed by results in fields less familiar to them than their own, and among these very many fields have not been covered here.

On the lighter side of the School, the participants were treated to a sparkling account of "The Golden Roman Days of Neutron Physics" given by Bruno Maximovitch Pontecorvo. It described the exciting personality of Enrico Fermi and revealed details of the life of Ettore Majorana; details not known to the general public. A quotation by Fermi retained our attention: "The work of a theorist, if it should have any sense, should be of extremely high quality. In this respect, we do not need more theorists than, say, egyptologists. Physicists with less than exceeding capabilities should do experimental work. For instance, measuring the density of all materials on earth is a useful work which does not require much insight."

How things have changed since! What would he say today?

N. Cindro, Zagreb, C. Kalbach, Saclay. 